\title{
Keeping current with regulations and requirements
}

\author{
B. Taylor Bennett \& Matthew R. Bailey
}

At a recent webinar hosted by the National Association for Biomedical Research, Drs. Elizabeth Meeks and William Stokes of the United States Department of Agriculture (USDA) answered approximately 50 questions submitted by participants. This article addresses several of those questions because they come up frequently, provide an opportunity to discuss a new issue, or are fundamental to the understanding of the regulatory process. This article has been reviewed by the USDA representatives and will attempt to provide additional clarification to the webinar responses.

\section{The IACUC authority to prescribe methods of research}

Is there language in the Animal Welfare Act $(\text { AWA })^{1}$ or the Animal Welfare Regulations (AWR) ${ }^{2}$ that would permit the Institutional Animal Care and Use Committee (IACUC) to prescribe methods or set standards for the design, performance or conduct of actual research? The simple answer to that question is yes, and in fact Congress was very specific about its intent in this regard as demonstrated by the language in the AWA.

Section 2143(a) of the AWA directs the Secretary of Agriculture to promulgate minimum standards "to govern the humane handling, care, treatment, and transportation of animals..." Additional language in this section enjoins the Secretary from promulgating "rules, regulations, or orders with regard to the design, outlines, or guidelines of actual research or experimentation by a research facility as determined by such research facility" except when such rules are consistent with the requirements of paragraph (3), sections (A) and (C)(ii) through (v), as well as paragraph (7).

National Association for Biomedical Research, Washington, DC. Correspondence should be addressed to B.T.B. (btbdvm@yahoo.com).
Paragraph (3)(A) addresses what takes place during experimental procedures to ensure the minimization of animal pain and distress through adequate veterinary care with "...the appropriate use of anesthetic, analgesic, tranquilizing drugs, or euthanasia." Paragraph (3)(C)(ii-v) addresses what must be done when a practice could cause pain to an animal, such as using tranquilizers, analgesics and anesthetics; providing pre- and postsurgical care, requiring the use of anesthetics when paralytics are used; and requiring that when tranquilizers, anesthetics, analgesics or euthanasia are to be withheld for scientific necessity that they are only withheld for the minimum period of time needed. Paragraph (7) addresses the language found in the annual report (APHIS Form 7023) where the Institutional Official provides assurance that professionally acceptable standards for the care, treatment and use of animals were followed during actual research.

Of note is that the requirements of paragraph (3)(B) are not included in this exception. Part (B) requires that investigators consider alternatives to procedures likely to cause pain and distress. Thus, when it comes to the use of alternatives, the regulations, and therefore the IACUC, cannot dictate "design, outlines, or guidelines of actual research or experimentation."

The regulations which established the IACUC and detailed its duties and responsibilities contain a prohibition for dictating methods of research unless doing so is provided for in the AWA and the AWR. Given the language in the AWA, the IACUC can dictate research methods as they relate to the provision of adequate veterinary care during the actual conduct of research to minimize pain and distress, unless withholding such care is scientifically justified and only withheld for the minimum time necessary, but cannot dictate the specific alternatives to be used if a research procedure may cause pain or distress.

\section{Assuring a quorum}

The AWA defines a quorum as "a majority of the members of the Committee" and requires a quorum for all formal actions of an IACUC. $\$ 2.31(\mathrm{~d})(2)$ of the AWR provides additional guidance for when full committee review is requested and a member of the committee has a conflicting interest, such as personal involvement in the proposed activity. The conflicted member may not participate in the review and approval of the activity except to provide information, and specifically cannot contribute to the constitution of a quorum. Conflicts of interest that may arise during full committee review should be identified in advance of an IACUC meeting to ensure there will be a quorum to conduct business if conflicted members are not able to participate.

\section{Oversight of the care and use of client- owned animals}

Whether client-owned animals used in clinical trials or for teaching purposes are covered by the regulatory oversight of the AWR has become a frequently asked question. Client-owned animals used in clinical trials in the context of a veterinary-clientpatient relationship are not regulated under the AWA; therefore a protocol or IACUC approval for these activities is not required. Animals owned by a shelter or pound that are receiving veterinary services, such as spays or neuters, are also considered to have a veterinary-client-patient relationship. How an institution provides internal oversight of client-owned animals is up to the institution, but if they elect to have the IACUC provide the oversight, the animals involved should not be included in the annual report (APHIS Form 7023) because they are not owned and maintained by the facility.

1. Animal Welfare Act, PL 89-544 as amended.

2. Animal Welfare Act regulations. 9 CFR, Chapter I, Subchapter A, Parts 1-3. 